\title{
Principi descrittivi ed esplicativi nel De Lingua Latina di Varrone
}

\author{
Viti, Carlotta
}

Posted at the Zurich Open Repository and Archive, University of Zurich ZORA URL: https://doi.org/10.5167/uzh-129928

Book Section

Published Version

Originally published at:

Viti, Carlotta (2016). Principi descrittivi ed esplicativi nel De Lingua Latina di Varrone. In: Poccetti, Paolo. Latinitatis Rationes. Descriptive and Historical Accounts for the Latin Language. Berlin: de Gruyter, 780-797. 


\section{Carlotta Viti \\ 48 Principi descrittivi ed esplicativi nel De lingua latina di Varrone}

\section{Introduzione}

Il presente articolo discute alcuni problemi del De Lingua Latina di Varrone, il più antico esempio di riflessione linguistica a Roma, le cui parti a noi pervenute sono dedicate a temi di etimologia (impositio vocabulorum, trattata nei libri V-VII) e di morfologia (declinatio vocabulorum, nei libri VIII-X). ${ }^{1}$ Nonostante Varrone sia stato altamente apprezzato nell'antichità per le sue ampie conoscenze e la sua moltitudine di interessi, in epoca moderna egli viene solitamente trascurato o analizzato con fini solamente storico-documentari. Le sue etimologie, infatti, sono ritenute solitamente errate, in quanto Volksetymologien in opposizione a gelehrte Etymologien (cf. Pisani 1967; Schmitt 1977; Malkiel 1993; Belardi 2002), e anche il dibattito fra analogia e anomalia presentato nella parte morfologica del De Lingua Latina è stata talvolta considerato una sua invenzione letteraria priva di valore storico (Fehling 1956, 1957).

Noi riteniamo invece che il De Lingua Latina di Varrone contenga numerosi motivi di interesse anche alla luce della teoria linguistica moderna. Innanzitutto, poiché morfologia ed etimologia sono gli ambiti linguistici maggiormente coltivati nel mondo classico, questo testo ci fornisce uno sguardo privilegiato sui principi della riflessione grammaticale antica. In secondo luogo, anche le sue etimologie errate possono ricevere una motivazione se osservate nel loro più ampio contesto culturale, come giustamente osservato da Katz (2010) nel suo articolo sull'etimologia nel mondo classico e medievale in genere, con particolare attenzione a Isidoro di Siviglia. Vedremo che la tradizione grammatica-

1 Dell'opera originale in venticinque libri sono andati perdute non solo l'introduzione (libro I) e la prima parte della discussione etimologica (libri II-IV), ma anche la parte finale della morfologia (libri XI-XIII) e l'intera sezione dedicata a temi di sintassi e stile (ordo et figurae, libri XIV-XXV). E' opportuno sottolineare che una trattazione della sintassi è un elemento nuovo nella tradizione grammaticale greco-romana, che verrà pienamente sviluppata solo a

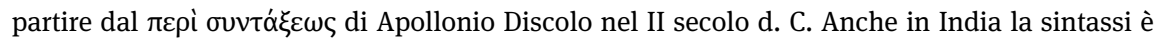
relativamente trascurata, almeno in confronto ad altre culture come quella semitica. Il Kitāb Sỉbawayhi (VIII secolo d. C.), su cui l'intera tradizione araba è basata, tratta principalmente morfologia, sintassi e lessico (mentre la fonetica è ritenuta un'appendice della morfologia), e il nome della sintassi (naḥw, lett. "direzione, metodo") significa anche "grammatica” in generale in arabo (cf. Cardona 1994; Fleisch 1994; Matthews 1994; Versteegh 1997). 
le antica si basava su un diverso concetto di descrizione, di classificazione e di spiegazione linguistica e più in generale epistemologica, che implica un ragionamento di tipo analogico, e che qui confronteremo con posizioni successive della filosofia del linguaggio e della filosofia della scienza.

\section{La parte etimologica del De Lingua Latina}

Le etimologie di Varrone presentate nei libri V-VII del De Lingua Latina sono talvolta corrette e talvolta errate alla luce della pratica etimologica moderna basata su regolari corrispondenze fonetiche. In V 68, ad esempio, Varrone cerca di spiegare il significato dei nomi del sole e della luna: sol vel quod ita Sabini, vel quod solus ita lucet, ut ex eo deo dies sit. Luna, vel quod sola lucet noctu. Mentre la connessione etimologica fra luna e la radice di luceo risulta sostanzialmente corretta, quella fra sol e solus "solo" certamente non lo è: Lat. sol solis deriva da un tema maschile e anficinetico (NOM * $s e ́ h_{2}-w o ̄ l$ vs. GEN $s h_{2}$-ul-és), che in proto-indo-europeo era in competizione con il tema neutro e proterocinetico NOM ${ }^{\star} s e ́ h_{2}-w l$ vs. GEN ${ }^{*} s h_{2}$-wén-s (Aw. huuarā vs. $x^{u} \bar{\partial} n g$ ). Le etimologie sbagliate come quella del sole prevalgono nel De Lingua Latina sulle etimologie giuste come quella della luna, cosa che, come si è detto, ha portato al giudizio negativo in toto su Varrone.

Tali errori derivano dal fatto che Varrone si lascia guidare nella comparazione lessicale da indiscriminate aggiunte, omissioni o mutamenti di segmenti fonetici: litterarum enim fit demptione aut additione et propter earum traiectionem aut commutationem, item syllabarum productione aut correptione, denique adiectione aut detrectione (V 6). Allo stesso modo, in VII 1 Varrone afferma che non devono essere rimproverati coloro che nell'esaminare una parola "aggiungono o tolgono una lettera" (litteram adiciunt aut demunt) - pratica che è stata definitivamente superata dall'etimologia almeno a partire dai Neogrammatici. Inoltre Varrone ritiene possibile che una stessa parola possa avere più di una etimologia, cosa oggi rifiutata tranne che nel caso di étymologie croisée identificato da Vendryes (1955). ${ }^{2}$

2 Nella cosiddetta etymologie croisée una parola A, con una determinata etimologia B, viene sentita dal parlante sincronicamente vicina ad un'altra parola con una differente etimologia $\mathrm{C}$, che arriva a influenzare la forma di A. Sebbene anche in questo caso abbiamo a che fare con un'etimologia popolare, essa ha delle conseguenze formali, per cui davvero la parola A deve essere spiegata in relazione a due etimologie diverse. Occorre inoltre ricordare che, se la connessione con più entità lessicali è solitamente inadeguata per l'etimologia scientifica praticata nell'indoeuropeistica, essa è invece apprezzata da un punto di vista storiografico e filosoficoletterario per la sua capacità evocativa di molteplici immagini. Come afferma De Bello (2007: 
Pur senza mettere in discussione i risultati della ricerca etimologica dei Neogrammatici, che ha portato alla stesura di celebri dizionari etimologici quali il Walde \& Pokorny (1927-32), ad esempio, vogliamo tuttavia enfatizzare alcuni spunti dell'etimologia del De Lingua Latina che possono essere interessanti, e talvolta perfino anticipatori, per la riflessione linguistica moderna.

Innanzitutto, quando Varrone attribuisce ad una parola più di una etimologia possibile, egli in genere discute differenti ipotesi, il che rivela un atteggiamento critico sulle fonti. In V 43, ad esempio, Varrone esamina il nome Aventinus, che come molti toponimi presenta una etimologia dibattuta (Aventinum aliquot de causis dicunt): per alcuni esso deriva da aves "uccelli" che vi si dirigono dal fiume Tevere, per altri dal nome del re Aventino che lì fu sepolto, per altri ancora da adventus "che viene" o da advectus "portato" (dall'acqua); quest'ultima etimologia è quella preferita da Varrone, in quanto l'Aventino era in passato separato da correnti o paludi dalle località adiacenti. Indipendentemente dal valore dell'etimologia proposta, Varrone non accetta semplicemente le ipotesi tradizionali, bensì le passa al vaglio anche considerando le proprietà fisiche del referente, come in questo caso la posizione geografica dell'Aventino. La ponderazione delle varie proposte etimologiche rispecchia la pratica del $\mathrm{di}$ sputare in utramque partem, che si farà più evidente nella parte morfologica.

In secondo luogo, Varrone è consapevole che fattori interni ed esterni possono avere effetti diversi sul lessico e che, di conseguenza, le parole straniere non possono essere analizzate alla stessa stregua di quelle native. Celebre è la sua tripartizione del lessico in V 10: igitur quoniam in haec sunt tripertita verba, quae sunt aut nostra aut aliena aut oblivia, de nostris dicam cur sint, de alienis unde sint, de obliviis relinquam. Una vera motivazione etimologica (cur), che implica il rapporto paradigmatico con altri lessemi, si può avere solo delle parole indigene. Allo stesso modo in $\mathrm{V} 77$, trattando i nomi degli animali acquatici, Varrone afferma che essi sono talvolta nativi e talvolta stranieri (partim sunt

42-43), "both etymology and allegory are used with reference either to a heuristic method be it allegoresis or a scientific reconstruction of etymological roots - or to instances of such methods in specific texts [...] That is why etymology and allegory straddle the jurisdictions of rhetoric, where they act as recognizable figurae, where they identify genres or themes; and of literary criticism, where they inspire and uphold interpretative forays. Finally, since both allegory and etymology make pronouncements about presumably inaccessible, or poorly accessible, origins (etyma or alloi that with disparate aims they set out to unravel), it seems that they grant or guard access to privileged knowledge, initiatory or not; that they function at once synchronically, yielding vibrant tranches de vie, and/or diachronically, tracing genealogical and 'original' connections; and that they operate on multiple, more or less correlated, layers or levels". Similmente, nella tradizione lessicografica antico- e medio-indiana viene praticata "l'étymologisation multiple du terme" (Balbir 1991: 130). 
vernacula, partim peregrina), e come tali necessitano anche di una trattazione diversa. Occorre sottolineare che la presa in considerazione di parole con un'origine diversa dal latino o dal greco è rara nella Roma repubblicana, mentre ha varie attestazioni nel De Lingua Latina, anche al di là della sezione etimologica. In VIII 65 Varrone si preoccupa della resa latina di parole straniere, cosa più o meno difficile a seconda della loro provenienza, e afferma che, mentre parole del punico e dell'antico egizio presentano una sola forma, poiché tali lingue non hanno casi, parole del celtico presentano una maggiore variazione. Anche parole greche possono essere più o meno acclimatate nella morfologia latina: in X 71 Varrone sostiene che alcuni nomi propri possono avere un accusativo plurale come Bacchidēs/Chrysidēs, come Bacchidĕs/Chrysiděs o come Bacchidăs/Chrysidăs. Sebbene tutte queste forme siano in uso, Varrone preferisce quelle intermedie, che presentano il timbro vocalico $e$ del latino e la quantità vocalica breve del greco, mentre le forme in $e$ lunga sono troppo distanti dal loro modello greco e quelle in $a$ breve sono troppo distanti dalla loro replica latina.

Oltre ai fattori diatopici, Varrone prende talvolta in considerazione anche quelli diacronici - cosa anch'essa piuttosto rara nell'antichità, dove l'etimologia ha una dimensione sostanzialmente sincronica, come vedremo in seguito. Già all'inizio del V libro Varrone evidenzia possibili effetti del mutamento linguistico nel tempo: quae ideo sunt obscuriora, quod neque omnis impositio verborum exstat, quod vetustas quasdam delevit, nec quae exstat sine mendo omnis imposita, nec quae recte est imposita, cuncta manet [...] multa verba aliud nunc ostendunt, aliud ante significabant, ut hostis: nam tum eo verbo dicebant peregrinum qui suis legibus uteretur, nunc dicunt eum quem tum dicebant perduellem (V 3). In questo senso, afferma Varrone, l'effetto distruttivo del tempo sul lessico non è diverso da quello sull'uomo e sulla natura (vetustas pauca non depravat, multa tollit), e paragona il mutamento linguistico al processo della vecchiaia, secondo una metafora biologica che avrà fortuna a partire da Schleicher (1873). La restituzione della forma o del significato originari è particolarmente difficile per la parole usate dai poeti, trattate nel VII libro, che talvolta sono affette da una repens ruina (VII 1), e nelle quali latent multa (VII 2). In questo modo Varrone ci tramanda parole o versi del latino arcaico che altrimenti sarebbero perduti.

Nella ricostruzione del valore originario di una parola, Varrone si serve spesso del confronto con lessemi appartenenti allo stesso livello paradigmatico. In V 13, per esempio, egli afferma che la relazione fra le parole consiste di molti membri (multa societas verborum), e in VIII 4 torna a fare un paragone con il mondo degli uomini: come fra gli uomini vi sono certe relazioni di parentela, così è fra le parole (ut in hominibus quaedam sunt agnationes ac gentilita- 
tes, sic in verbis). Il paragone con parole derivate dalla stessa radice gli permette di stabilire meglio un'etimologia, anticipando in questo modo alcuni principi della Wortfeldtheorie.

Più in generale, l'elemento dell'etimologia varroniana che ancora oggi si rivela valido è la sua fondamentale interdisciplinarietà. Ancora in V 13 Varrone annuncia che, pur trattando i nomi di luogo, non si esimerà di affrontare parole con significato diverso che abbiano con essi una certa "relazione" (cognatio), e lo afferma usando una metafora tratta dal mondo vegetale: sed qua cognatio eius erit verbi quae radices egerit extra fines suas, persequemur. Saepe enim ad limitem arboris radices sub vicini prodierunt segetem. In questo modo Varrone fa spesso riferimento a dati di carattere storico, antiquario o letterario nel perseguire un'etimologia, cosa che gli è permessa dalla sua formazione enciclopedica e non strettamente grammaticale. Un tale eclettismo è oggi raccomandato dagli studi più recenti di etimologia e di semantica, che intende supplementare le nude corrispondenze delle Lautgesetze dei Neogrammatici con una più ampia attenzione alle connotazioni socio-culturali di una parola. Così scrive Durkin nel suo manuale di etimologia: "etymology can be a very demanding area of linguistic research, drawing on many different aspects of linguistics. It also draws at time on a good deal of non-linguistic information, about the transmission of texts or other sources of data, or about developments in social or cultural history. For this very reason it can also be extremely rewarding. Few areas of study offer points of contact with so many other fields" (2009: 287). Allo stesso modo si esprime Evans: "Semantic typology thus lies directly on the fault lines between psychology, neuroscience, anthropology, the natural sciences, and general linguistics" (2011: 505).

\section{La parte morfologica del De Lingua Latina}

Spunti interessanti emergono anche nella seconda parte del De Lingua Latina, quella dedicata alla morfologia, dove Varrone mette a punto tecniche sia descrittive che esplicative della sua lingua. Una descrizione è perseguita mediante una classificazione del lessico in base a criteri formali, secondo cui la parola, vista come unità minima del lessico (X 77), ${ }^{3}$ viene distinta a seconda che sia

3 L'unità del morfema non è stata individuata né nella tradizione grammaticale greco-romana né in quella indiana, in cui tuttavia venivano riconosciute le unità morfologiche della radice (dhātu), dell'affisso (pratyaya) e della desinenza (anta, vibhakti). Possiamo mettere in relazione questa apparente lacuna con l'osservazione, corrente nei testi di morfologia, per cui il morfema è soprattutto evidente nelle lingua agglutinanti ("the whole notion of morpheme works best when each word is easily divisible into one or more discrete morphs", Bauer 2003: 110). 
declinabile, nel qual caso abbiamo il genus fecundum, o indeclinabile, nel genus sterile: duo enim genera verborum, unum fecundum, quod declinando multas ex se parit disparilis formas, ut est lego legi legam, sic alia, alterum genus sterile, quod ex se parit nihil, ut est et iam vix cras magis cur (VIII 9). All'interno del genus fecundum, poi, Varrone identifica varie relazioni di somiglianza o di differenza fra le forme, che possono essere classificate in quattro tipi: 1) $a b$ similibus similia, come dalle forme analoghe bonus e malus si ricavano forme altrettanto analoghe quali bonum e malum; 2) ab similibus dissimilia, come dagli analoghi nominativi lupus e lepus si hanno i differenti dativi lupo e lepori; 3) ab dissimilibus dissimilia, come i differenti nominativi Priamus e Paris danno luogo a dativi altrettanto differenti quali Priamo e Pari; 4) ab dissimilibus similia, ad es. Iuppiter e ovis che però hanno dativi analoghi quali Iovi e ovi (VIII 34). Secondo Varrone, la somiglianza non si deve cercare né fra gli indeclinabili (X 79) né fra i difettivi (X 82), ma solo in parole di uso corrente che siano provviste di una flessione completa. In questo modo Varrone cerca di stabilire dei comparanda grammaticali (X 83), che possono essere utili anche per l'etimologia stabilita dal metodo comparativo, il cui principio primo è secondo Watkins (1990: 300) che you have to know what to compare.

Una volta classificato il lessico della sua lingua, Varrone esprime anche la consapevolezza che non tutte le forme possibili sono altrettanto ugualmente accettabili, e questo lo porta a considerare l'usus e la consuetudo come i fattori essenziali che devono guidare il parlante, e che sono addirittura più importanti della regolarità, nel convincimento che potius consuetudinem quam analogias dominari (VIII 79). L'uso può spiegare la difettività nella flessione o nella derivazione: mentre, ad esempio, la distinzione fra l'esemplare maschio e femmina del cavallo è rilevante nel mondo contadino e viene quindi lessicalizzata nella coppia equus ed equa, non vi è una forma femminile ${ }^{*}$ corva corrispondente a corvus, perché una tale differenza risulta inutile (IX 56). Allo stesso tempo, se vi è un mutamento nel dominio esperienziale, se ne può prendere atto anche nel lessico, per cui in un primo tempo il nome columba veniva usato indipendentemente dal sesso del referente, mentre in seguito alla sua domesticazione si è avuta in latino la distinzione fra columbus e columba. Il grado di accettabilità di una struttura linguistica, inoltre, dipende anche chi la usa: la libertà dell'oratore, ad esempio, è minore di quella del poeta, il quale può transilire lineas impune (IX 5). In ogni caso il parlante deve mirare ad una comunicazione efficace, il che è possibile solo se sarà chiara e breve (omnis oratio cum debeat dirigi ad utilitatem, ad quam tum denique pervenit, si est aperta et brevis,

In quest'ottica, possiamo ipotizzare che la mancata individuazione del morfema da parte dei grammatici antichi sia dovuta all'articolata morfologia fusionale delle loro lingue. 
VIII 26). In principio, per una tale comunicazione non c'è bisogno di regolarità, bensì di un uso consolidato. In pratica, tuttavia, uso e regolarità sono strettamente associati (consuetudo et analogia coniunctiores sunt inter se quam iei credunt, IX 2), e questo ci porta al punto seguente, ossia all'equilibrio fra storage e processing.

La competizione funzionale fra l'immagazzinamento di dati nella memoria (storage) e l'elaborazione e produzione di nuove forme secondo regole già acquisite (processing) viene anticipata dalla contrapposizione varroniana fra lessico e grammatica. Egli afferma che la flessione è cosa utile e necessaria, e che pertanto le sue risorse debbono essere sfruttate il più possibile nelle lingue, anche al di là del latino (declinatio inducta in sermones non solum Latinos, sed omnium hominum utili et necessaria de causa, VIII 3), poiché senza flessione non potremmo padroneggiare un così gran numero di parole, né potremmo riconoscere la relazione intercorrente fra le parole che abbiamo già imparato. Di conseguenza, mentre la declinatio deve essere estesa, come una propago o come un rivus, l'impositio di parole nuove, simile ad una fons, deve essere limitata (impositicia nomina esse voluerunt quam paucissima, quo citius ediscere possent, declinata quam plurima, quo facilius omnes quibus ad usum opus esset dicerent, VIII 5). ${ }^{4}$

Come nell'etimologia Varrone prende spesso in considerazione differenti opinioni, così nella morfologia egli imposta la sua analisi in maniera dialettica, con riferimento al celebre dibattito fra gli analogisti della scuola di Alessandria e gli anomalisti della scuola di Pergamo. Molto si è discusso nella letteratura sulla veridicità di un tale dibattito e sull'originalità o meno di Varrone nel riportarlo (cf. Dahlmann 1932; Taylor 1975; Pfaffel 1981; Ax 2000: 140a ss). Se guardiamo all'organizzazione del testo, possiamo osservare che alcuni problemi sollevati nell'ottavo libro, a favore dell'Anomalia, trovano una soluzione nel libro nono, a favore dell'Analogia, con tutta una serie di richiami intertestuali che, indipendentemente dalle fonti utilizzate, dimostrano quantomeno una rielaborazione originale del materiale da parte di Varrone. ${ }^{5}$

4 Ciò è correlato alla distinzione varroniana fra declinatio voluntaria e declinatio naturalis: la prima consiste nello stabilire nuovi nomi, mentre la seconda è la loro flessione regolare secondo i principi della grammatica (cf. VIII 22, IX 34, X 15). Si noti il senso ampio del termine declinatio, che include non solo sia declinazione che coniugazione, ma anche la derivazione accanto alla flessione. Così in VIII 52 Varrone differenzia quattro tipi di declinatio: la flessione come da equus ad equum, la derivazione come da equus a equile, il comparativo come da albus ad albior, e il diminutivo come da cista a cistula.

5 Non dobbiamo dimenticare che il problema dell'originalità e dell'imitazione ( $\mu$ í $\mu$ $\sigma \varsigma$ ) era sentito in modo molto diverso rispetto al nostro nell'antichità, quando il collocarsi in una tradizione ben definita dall'auctoritas e il mostrare somiglianza (عíkúv) con un modello, eventualmente raffinando alcuni dettagli, era visto fondamentalmente in maniera positiva. In ogni 
Primo, in VIII 28 ss Varrone scrive che nei nostri oggetti di uso quotidiano noi cerchiamo l'utile, e non la somiglianza (in his nostrumst utilitatem quaerere, non similitudinem), per cui nel vestiario di un uomo la toga è diversa dalla tunica, e in quello di una donna la stola è diversa dal pallium. Così nelle abitazioni la camera da letto (cubiculum) è diversa dalla stalla (equile), e gli spazi usati nell'inverno o nell'estate sono anch'essi diversamente forniti di porte e finestre. Di conseguenza, ne deduce Varrone, una mancata somiglianza non sarà da ripudiare neppure nel parlare. In IX $48 \mathrm{ss,} \mathrm{tuttavia,} \mathrm{si} \mathrm{osserva} \mathrm{che} \mathrm{la}$ tunica di un uomo è simile nella sua forma al corpo di un uomo, la stola di una donna al corpo di una donna, e così anche nell'uso delle suppellettili la somiglianza o l'armonia sono spesso ricercate. Secondo, in VIII 30 Varrone sostiene che, se comunque le differenze sembrano prevalere nel mondo, esse non debbono essere neppure escluse dal parlare. D’altra parte, la prevalenza della varietà non è affatto cosa scontata, anzi la natura tutta sembra seguire principi di regolarità: quae enim est pars mundi quae non innumerabiles habeat analogias? Caelum an mare an terra, quae in his? (IX 23) Terzo, in VIII 32 si osserva che oggetti differenti (disparia) sono solitamente piacevoli (iucunda), per cui gli uomini cercano di non vestirsi tutti uguali o di non arredare in modo identico le loro case e le loro stanze. D’altro lato, si dice in IX 46, se vi è piacere nella varietà, allora vi è un piacere ancora maggiore nell'armonia, o nel fatto che alcuni oggetti siano simili fra loro. Quarto, se la regolarità non è completa, non si può dire che essa sussista, così come il fatto che un Etiope abbia i denti bianchi non è sufficiente per dire che egli è bianco (VIII 38). Nondimeno, si ribatte in IX 78, se una statua manca della testa, questo non significa che le altri parti del corpo non possano essere regolari e armoniche.

In questo dibattito Varrone ha una posizione intermedia: per lui sia l'analogia sia l'anomalia debbono essere seguite, in quanto entrambe presiedono a diversi ambiti della lingua, l'analogia alla declinatio naturalis, ossia alla flessione, e l'anomalia alla declinatio voluntaria, ossia la formazione di un lessema (VIII 23). Ciò ricorda l'atteggiamento aporetico dei primi dialoghi platonici, come il Cratilo, anch'esso dedicato a problemi linguistici e in particolare alla

caso, la presenza di un dibattito già sviluppato su analogia e anomalia sembra emergere

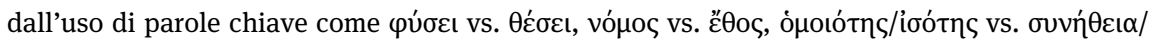

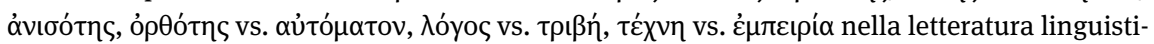
co-filosofica a partire dal Cratilo di Platone, cf. Lersch (1838: $4 \mathrm{ff}$.). Tali temi ricorrono anche al di là del dominio IE, ad esempio nel mondo arabo, dove la polemica fra analogia e anomalia coinvolge, in maniera non sempre dipendente dalle fonti greche, le due città di Basra e Kufa, rispettivamente, e dove l'analogia (al-qiyās) è un importante principio di analisi grammaticale (cf. Baalbaki 2013: 92ss). 
correttezza dei nomi. Avere una posizione intermedia, tuttavia, non significa essere equidistante dalle due opinioni: come Platone è più vicino alla posizio-

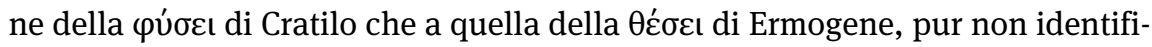
candosi pienamente con nessuna delle due, così Varrone sembra essere più vicino all'Analogia che all'Anomalia ("er ist jedoch eher als gemäßigter Analogist zu bezeichnen, auch wenn der Anomalie ein Platz bei der Wortschöpfung und bei der Derivation eingeräumt wird ", Jungen \& Lohnstein 2006: 41). Nel disputare in utramque partem Varrone mostra la dialetticità della tradizione platonica e, al tempo stesso, la sistematicità di Aristotele, il quale invece si fa avvocato della $\theta \dot{\varepsilon} \sigma \varepsilon \iota$ e del convenzionalismo linguistico.

\section{Denotazione, descrizione e spiegazione nel De Lingua Latina}

Nella sua opposizione fra l'uso a qua re e quello in qua re (V 2) Varrone distingue fra l'etimologia vera e propria, che mira a stabilire "da dove" (unde) una parola sia originata, e la semantica, che invece ha come oggetto il significato di una parola e a cosa essa si riferisca. L'etimologia, a sua volta, può essere rappresentata dalla relazione temporale $B a b A$ oppure dalla relazione causale $B$ quod A (cf. Pfaffel 1981). E' importante sottolineare che le due dimensioni possono essere più o meno distinte a seconda che il significato lessicale sia sincronicamente opaco o trasparente in una lingua. Lo stesso vale per la distinzione, corrente negli studi di semantica, fra "denotazione" e "descrizione": mentre la prima indica semplicemente l'oggetto a cui una parola si riferisce, la seconda riguarda il modo in cui certe caratteristiche del referente invece che altre vengono catturate dalla lessicalizzazione (Pisani 1967; Lyons 1977: 174 ss). La parola latina homo e il suo corrispondente arabo insān, ad esempio, hanno entrambi come denotazione l'essere umano, ma lo descrivono in maniera diversa, homo come "terrestre" (cf. humus), insān come "compagno", dalla radice anisa "essere compagno, sociale, amichevole” (cf. anis "vicino, intimo, amico").

La descrizione, così come l'uso varroniano a qua re, può essere vista già come un tipo di spiegazione, in quanto fornisce un razionale al di là di quella che a prima vista sembra una sequenza fonica arbitraria. Lo stesso De Saussure (1916), dopo aver stabilito l'arbitrarietà del segno, ha anche ammesso la possibilità di una motivazione parziale, in caso una parola possa essere ricondotta ad un'altra tramite composizione o derivazione. Anche in questo caso, l'efficacia di una descrizione sarà maggiore se la parola in questione mantiene una sua trasparenza a livello sincronico e presenta potenziali connessioni con altri 
lessemi corradicali all'interno di un sistema linguistico. Nelle lingue semitiche la trasparenza di una parola, basata sull'individuazione della radice, è più elevata che nelle lingue indoeuropee, così come nelle lingue indoeropee antiche la corradicalità era più evidente che in quelle moderne. I quattuor explanandi gradus di Varrone (V 7), che secondo Langendoen (1966) possono rappresentare il passaggio dall'adeguatezza osservativa a quella descrittiva fino a quella esplicativa nel senso di Chomsky, presuppongono in realtà differenti gradi di trasparenza sincronica. Il livello più basso, tipico del populus, è quello di parole complesse che possono essere ricondotte a parole semplici ancora in uso, come il composto argentifodinae "miniere d'argento". Il secondo livello, della grammatica antiqua, riguarda parole obsolete che sono state appunto preservate dai grammatici. Il terzo gradus explanandi, l'ultimo a cui Varrone intende arrivare, è quello dei filosofi, i soli in grado di comprendere il significato di parole sincronicamente opache come oppidum, e il quarto livello è quello dei sacerdoti (adytum et initia regis). ${ }^{6}$ La spiegazione, quindi, diventa più difficile a mano a mano che la parola risulta isolata nel lessico e priva di trasparenza sincronica. I due concetti della denotazione e della descrizione, tradizionalmente presentati sullo stesso piano, possono avere un legame diacronico l'un con l'altro, quando un'espressione descrittiva trasparente nel sistema diventa col tempo opaca e viene vista come arbitraria denotazione.

Possiamo ipotizzare che è proprio la frequente trasparenza della parola a giustificare la pratica dell'etimologia sincronica corrente nel mondo antico e scorretta da un punto di vista storico: poiché allora una parola poteva essere in molti casi accostata a suoi corradicali in modo chiaro, questo procedimento viene semplicemente esteso agli altri casi, in cui invece la somiglianza era solo apparente. ${ }^{7}$

Seppur errata da un punto di vista scientifico, quindi, l'etimologia antica può essere meglio compresa se consideriamo il tipo di spiegazione ricercato nell'antichità, che è molto diverso da quello oggi riconosciuto nella filosofia del linguaggio (Leiss 2009; Newen \& Schrenk 2008; Bertram 2011) e nella filo-

6 Come osserva De Bello (2007: 85), "the quartus gradus etymologiae is a form of initiation described in hieratic terms (as adytum; as initia regis) that go well beyond grammar or rhetoric". In questo stadio De Bello vede una manifestazione dell'allegoria, "as a way to accessing metaphysical (i.e., non-technical) truths" (p. 94).

7 Chiarezza e rapporti sincronici sono al centro dell'analisi anche nella parte morfologica del De Lingua Latina. In X 56, ad esempio, Varrone afferma che si deve cominciare dal chiaro piuttosto che dal precedente (potius proficisci oportet ab eo quod apertius est quam ab eo quod prius est). Così la declinazione sarebbe più facile da inferire se si prende come punto di partenza il nominativo plurale o l'ablativo singolare invece che il nominativo singolare, in cui la consonante del tema spesso non è visibile con l'aggiunta della desinenza -s. 
sofia della scienza (Okasha 2002: 40 ff.; Curd et al. 2013: 647 ff.). Oggi si utilizza solitamente una spiegazione temporale o causale in linguistica storica, per cui un certo fenomeno $\mathrm{X}$ è tale in quanto causato da un'altra situazione $\mathrm{Y}$ ad esso precedente (explanatio ut prior eventus sive causa); è questa l'interpretazione di spiegazione etimologica stabilita dai Neogrammatici. In alternativa, soprattutto nelle scienze naturali, si considera $\mathrm{X}$ come il risultato di costituenti elementari a cui si perviene tramite un processo di astrazione e semplificazione (explanatio ut reductio). Nel mondo antico, invece, una spiegazione consisteva nel considerare $\mathrm{X}$ un exemplum di $\mathrm{Y}$ e nel mettere i due fenomeni in relazione analogica (explanatio ut translatio), oppure nel correlare un fenomeno incerto con un altro che sia più familiare, come afferma Quintiliano: eius (sc. analogiae) haec vis est, ut id quod dubium est ad aliquid simile de quo non quaeritur referat, et incerta certis probet (Inst. 1,6,4). Tale analogia viene basata fondamentalmente sulla trasparenza morfologica e sul rapporto di somiglianza nel corpo fonico della parola ("similitudo is the basis of any and all comparison and classification”, Taylor 1977: 314). ${ }^{8}$ Non è forse un caso che, anche a livello filologico, le parti a noi pervenute del De Lingua Latina riguardino l'etimologia e, in morfologia, il dibattito sulla validità dell'analogia: l'analogia, intesa da Varrone nel senso più ampio di regolarità, sistematicità e somiglianza ${ }^{9}$ fornisce la spiegazione agli interrogativi etimologici. Tutto ciò è molto diverso dal modo l'analogia è stata solitamente trattata nella linguistica moderna. De Saussure (1916), ad esempio, contrappone analogia ed etimologia popolare: mentre l'analogia è un "normale" meccanismo del mutamento linguistico, l'etimologia popolare sarebbe un "fenomeno patologico" (cf. Reichler-Béguelin 1995).

8 Taylor (1977) spiega che la somiglianza di Varrone è intesa come corrispondenza fra la figu$r a$, ossia la forma fonologica della parola, e la materia, il suo contenuto grammaticale, e analizza una tale corrispondenza secondo varie proporzioni di tipo matematico, per cui "words, in order to be classified together, must exhibit, in terms of their phonological form and morphological content, the same type of exact relationships as numbers in mathematical proportions." (p. 322; cf. anche Taylor 1996) Pur apprezzando la descrizione delle proporzioni soggiacenti alle classificazioni varroniane, pensiamo che non sia corretto enfatizzare il loro valore matematico. La somiglianza e l'analogia rappresentano un fondamentale processo cognitivo (cf. Best 1973; Esper 1973; Anttila 1977) di cui la matematica è solo una delle possibili rappresentazioni. Più rilevante nell'antichità sono probabilmente le sue implicazioni nella magia e nella divinazione (cf. Noegel 2010), per cui un simbolo equivale al suo referente extralinguistico.

9 Nonostante l'interpretazione lato sensu, l'analogia varroniana comprende solo una parte dell'analogia come è intesa oggi in linguistica storica, e cioè l'analogia proporzionale, e non il livellamento. La prima è una modificazione paradigmatica dovuta alla somiglianza con la forma di un altro paradigma, come quando Varrone interpreta il GEN.PL boverum, anziché boum, come dovuto all'analogia con iugerum (VIII 74). Il livellamento (levelling), invece, consiste nella perdita di distinzioni morfologiche all'interno dello stesso paradigma (cf. Hock 1991: 167 ff.), cosa meno evidente nella ricca morfologia nominale latina. 
Possiamo altresì ipotizzare che la frequente - e spesso sovraestesa - trasparenza lessicale è il motivo per cui gran parte dei filosofi e grammatici anti-

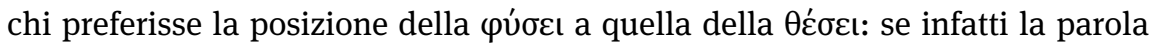
viene a descrivere il referente in maniera trasparente, si può facilmente pensare che essa rispecchi anche la sua natura e in qualche modo dica il vero. In quest'ottica, l'arbitrarietà à la De Saussure diventa inconcepibile: essa equivale a pensare che non vi sia una ratio nel mondo, e ciò va ben al di là dell'etimologia e della grammatica, fino a coinvolgere ogni genere letterario e perfino, nel mondo esterno, il rapporto dell'uomo con la storia e con gli dei. Basti pensare alla differente rappresentazione del mito nei diversi tragediografi greci. In Eschilo i mali dell'uomo trovano una loro spiegazione in una precedente colpa di lui o dei suoi antenati, e la punizione degli dei ha addirittura una funzione didattica, perché l'uomo può finalmente comprendere la colpa e cercare di redimersi, come succede ad Oreste che, dopo aver ucciso la madre, viene prima perseguitato dalle Erinni ma poi assolto di fronte al tribunale dell'Aeropago: in Eschilo vige il principio del $\delta ı \dot{\alpha} \pi \alpha ́ \theta$ os $\mu \alpha \dot{\theta} \theta$ os. Questa corrispondenza diretta fra colpa e punizione comincia ad incrinarsi in Sofocle. Edipo ha sì compiuto una colpa - la peggiore colpa immaginabile - uccidendo il padre e sposando sua madre Giocasta, ma non era consapevole dell'identità dei genitori: è quindi giusto che sia stato punito in maniera così terribile? Sofocle lancia il dubbio sulla validità della precedente tradizione. In Euripide, infine, non c'è sempre una colpa che possa giustificare i mali dell'uomo, anzi spesso l'infelicità umana è causata ingiustamente dagli dei. Ippolito non è affatto colpevole, ma non lo è neppure il padre Teseo che, credendo alle false accuse di Fedra, lo ha mandato in esilio, e neppure Fedra è colpevole, visto che l'amore per il figliastro le è stato trasmesso con l'inganno da Afrodite. In questo riconoscimento dell'ingiustizia dei mali sta la modernità di Euripide, la cui posizione è infatti assolutamente anomala nel pensiero antico. La civiltà antica si basa fondamentalmente sulla rispondenza fra colpa e punizione, così come sulla ricerca dell'uomo di propiziarsi il favore del proprio signore o degli dei per mezzo di doni - di ciò vi sono testimonianze infinite nei miti, nei rituali e nelle leggi non solo del mondo classico, ma anche delle civiltà del Vicino Oriente. L'idea che il nome rifletta la vera natura del referente va inquadrata, a nostro avviso, in questa visione generale di ricerca di un certo ordine nel mondo. Il parlare, infatti, veniva visto allo stesso modo delle altre attività umane: nel Cratilo

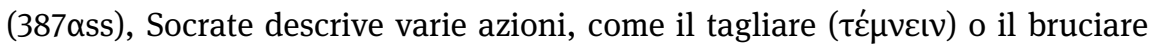

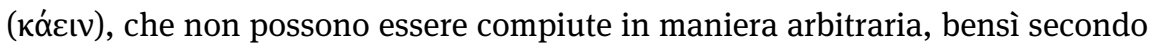
la natura delle cose da tagliare o da bruciare, e che richiedono al loro agente

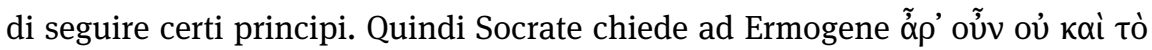

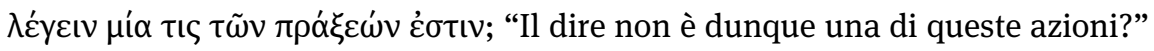


e, alla risposta affermativa di Ermogene, egli spiega come anche il "denomina-

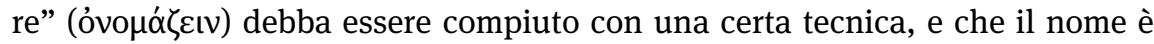
uno "strumento" (öpyavov), così come lo è la spola per il tessitore. In particolare, "il nome è uno strumento per insegnare e per distinguere l'essenza delle

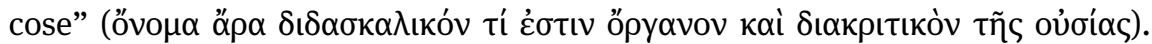
Anche se Socrate non accetterà pienamente neppure la posizione del naturalismo di Cratilo, che egli mira a raffinare nella seconda parte del dialogo, ${ }^{10}$ la confutazione del convenzionalismo di Ermogene avviene subito in maniera programmatica. Credere nell'arbitrarietà dei nomi, così come nell'arbitrarietà degli eventi del mondo sarebbe stato un pensiero "scandaloso" nell'antichità.

\section{Etimologia popolare e pensiero analogico nell'antichità}

La ricerca di una somiglianza sincronica fra le parole, con conseguente etimologia popolare, non è dovuta alla mancata conoscenza del mutamento linguistico o a una visione non scientifica della fonetica. Varrone è ben consapevole degli effetti della vetustas sul corpo fonico della parola, come si è visto in $\S 2$, e lo stesso vale per Yāska in India, che nel suo Nirukta spiega solo le parole divenute nella sua epoca obsolete. Allo stesso modo la fonetica, se trascurata in Grecia e a Roma, dove il suono viene spesso confuso con la sua rappresenta-

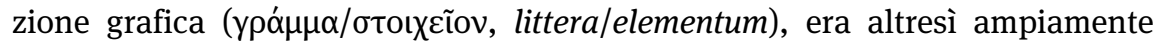
coltivata in India, in cui perfino il sandhi post-lessicale viene registrato, inizialmente con il fine pratico di permettere una lettura corretta degli testi religiosi (cf. Viti 2014). L'etimologia popolare dell'antichità è piuttosto basata su una differente concezione della parola, della lingua, e più in generale del mondo, per cui ogni espressione verbale è fondamentalmente iconica e porta in sé una motivazione, anche se non tutti, ma solo il saggio, il sacerdote o il filosofo, la possono comprendere.

Nel prendere atto di una trasparenza lessicale, al suono veniva assegnato un ruolo di primo piano. Nel chiedersi se la somiglianza fra parole si debba basare più sulla loro rappresentazione fonetica ( $a$ voce) oppure semantica ( $a$ significatione), Varrone predilige il suono (IX 40), e ciò va ben al di là delle parole onomatopeiche come upupa, cuculus, ulula, bubo, che derivano il loro

10 Il motivo per cui Socrate non accetta completamente il naturalismo di Cratilo, e per cui il dialogo rimane aporetico, è che la realtà secondo Platone è di per sé inconoscibile all'uomo e quello che noi percepiamo è soltanto apparenza (cf. Lallot 1991; Sedley 2003; Ademollo 2011). 
nome $a b$ suis vocibus (V 75). Poiché il dato formale viene tradizionalmente preferito a quello semantico anche nella tradizione dell'indoeuropeistica (Benveniste 1935: 148), ci possiamo chiedere in che cosa differisca l'interpretazione del suono nell'antichità e nella pratica etimologica moderna. Il maggiore fattore di differenza è che al suono stesso, prima che alla parola, veniva assegnato

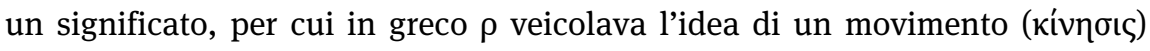

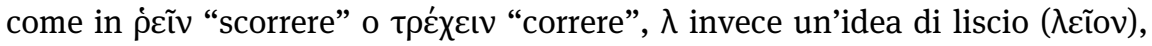

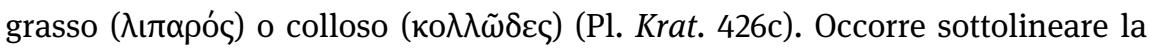
portata teorica di una tale concezione, in cui vengono meno non solo il rapporto arbitrario fra significante e significato, ma anche il principio della doppia articolazione, perché le unità minime del secondo livello di articolazione, i fonemi, vengono visti nel mondo antico come essi stessi portatori di significato, al contrario di quando presupposto dalla linguistica moderna. Il fonosimbolismo, considerato privo di valore scientifico da Meillet (1931: 21), aveva nell'antichità una suprema rilevanza nell'individuare corrispondenze lessicali e nel decifrare un ordine nell'apparente idiosincrasia del lessico. Per i grammatici indiani śabda è "suono", ma al tempo stesso può avere anche il valore di artha "significato" e può essere interpretato secondo i contesti come "parola" o “discorso” (cf. Coward \& Kunjunni Raja 1990: 5). Allitterazione e assonanza vengono ricercate in tutta la poesia orale antica, dagli inni vedici ai carmina latini, e non solo come tecnica mnemonica, ma anche come strategia per dotare il testo della magia del suono.

L'uso del suono, con le sue implicazioni magico-religiose, nello stabilire relazioni lessicali trova paralleli anche in altre culture non indoeuropee del mondo antico, dove la Volksetymologie e la Lautmalerei erano altrettanto comuni. Nell'antico Egitto abbiamo liste di parole ordinate tematicamente (onomastica) o foneticamente, nel caso inizino con la stessa consonante. Una di queste (testo 27 di Saqqara) elenca una serie di frasi in cui un certo uccello si trova su un certo albero, e il nome dell'uccello e quello dell'albero iniziano con la stessa consonante, es. pe hb hrr pe hbyn "l'ibis è sull'albero di ebano" (cf. Johnson 1994: 67). Tali allitterazioni suggeriscono etimologie popolari e giochi di parole, per cui il teonimo Horus, ad esempio, viene ricondotto per para-etimologia alla radice $h r$ "essere lontano", secondo la concezione di Horus come dio del cielo. Anche qui vige il principio del naturalismo nella denominazione:

The Ancient Egyptians believed that names were an integral part of an entity, designating its essence or a particular trait or feature, the basic concept being that the owner of a name became endowed with all the powers connected with it etymologically. The bearer of a name or epithet sometimes received it because he possessed certain qualities, or because of certain acts on his part; in some cases a 'name' may be invented specifically for single use in order to make a certain act possible. The 
awe with which the Ancient Egyptians regarded names, and the importance which they attributed to them, leave no doubt that they looked upon this kind of ad hoc word-play as etymologies in the strictly literal sense of the word.

(Gilula 1982: 259-60)

Similmente, in accadico i teonimi venivano spesso spiegati mediante etimologie popolari stabilite in base alla somiglianza dei suoni. Il nome della dea babilonese Zarpānītu, ad esempio, veniva considerato un composto di zēru "seme, progenie" e bānītu "creatrice”: Zarpānitu ša kīma šumišama bānât zēri "Zarpānītu che, secondo il suo nome, è creatrice di progenie." (Reiner 1994: 92; cf. anche Jacobsen 1974) L'adozione delle stesse procedure etimologiche in gran parte del mondo antico, indipendentemente dalla lingua, dall'arealità e anche dallo sviluppo di una tradizione grammaticale, suggerisce di interpretare queste etimologie popolari non come semplici errori o bizzarri accostamenti lessicali, bensì come l'espressione di una cultura in cui l'attenzione al suono e un pensiero analogico, portato a cogliere le somiglianze formali, avevano un ruolo diverso, molto più importante, che nella linguistica attuale. La conferma ci viene dalla ricerca antropologica condotta su comunità linguistiche che ancora oggi si servono di una trasmissione prevalentemente orale, in cui la parola assume un valore sacrale, che solo l'iniziato può comprendere, e che ha una vera e propria capacità di agire e di influenzare il corso degli eventi. E' questa la parole agissante che Nathan et Hounkpatin (1993: 796) identificano nella tradizione orale degli Yoruba, "la parole [...] considérée [...] comme principale véhicule de pouvoir - le pouvoir de détruire, le pouvoir de donner la vie, le pouvoir de modifier ce qui existe."

\section{Conclusioni}

In questo articolo abbiamo discusso alcuni problemi della filosofia del linguaggio nel mondo antico, con particolare attenzione al De Lingua Latina di Varrone. Varrone illustra bene le Fragestellungen dell'antica riflessione linguistica, ossia quale sia l'origine delle parole, il loro vero significato e il loro uso corretto, come si possa categorizzare il lessico della propria lingua secondo criteri formali o semantici, e con quali procedimenti si possano formare parole nuove. Abbiamo visto che il problema dell'etimologia popolare e degli accostamenti analogici nel mondo antico va affrontato in maniera olistica, con riferimento ad una diversa concezione della comunicazione, della conoscenza e del concetto stesso di "spiegazione". La spiegazione analogica di Varrone, anziché essere semplificemente frutto di un'errata valutazione dei dati linguistici, affonda le 
sue radici in una cultura orale e in un'antica practica magica e religiosa, evidente negli scritti di varie lingue indoeuropee e non, anche al di là del latino. Nella sua ricerca di iconicità, inoltre, essa si rivela vicina a certe rivalutazioni della motivazione linguistica, accanto all'arbitrarietà, portate avanti a partire da Haiman (1985) in campo funzionalista.

\section{Bibliografia}

Ademollo, F. 2011. The Cratylus of Plato, Cambridge, CUP.

Anttila, R. 1977. Analogy, The Hague, Mouton.

Ax, W. 2000. Lexis und Logos, Stuttgart, Steiner.

Baalbaki, R. 2013. Arabic linguistic tradition I: naḥw and șarf, in The Oxford Handbook of Arabic linguistics, ed. J. Owens, Oxford, OUP. 92-114.

Balbir, N. 1991: Le discours étymologique dans l'hétérodoxie indienne, in J.-P. Chambon/ G. Lüdi (eds.): 121-134.

Bauer, L. 2003². Introducing linguistic morphology, Edinburgh, Edinburgh University Press. Belardi, W. 2002. L'etimologia nella storia della cultura occidentale, Roma, Il Calamo. Benveniste, É. 1935. Origines de la formation des noms en indo-européen, Paris.

Bertram, G. 2011. Sprachphilosophie zur Einführung, Hamburg, Junius.

Best, K.-H. 1973. Probleme der Analogieforschung, München, Hüber.

Cardona, G. 1994. Indian linguistics, in G., Lepschy (ed.), Volume I. 25-60.

Chambon, J.-P./ Lüdi, G. (eds.) 1991. Discours étymologiques, Tübingen, Niemeyer.

Coward, H. and Kunjunni Raja, K. 1990. Encyclopaedia of Indian philosophy. The philosophy of the grammarians, Princeton, Princeton University Press.

Curd, M, Cover, J. and Pincock, Ch. (eds.). ${ }^{2} 2013$. Philosophy of science. The central issues, New York/London, Norton / Co.

Dahlmann, H. 1932. Varro und die hellenistische Sprachtheorie, Berlin, Weidmannsche Buchhandlung.

De Bello, D. 2007. Forgotten paths. Etymology and the allegorical mindset, Washington DC, The Catholic University of America Press.

Durkin, Ph. 2009. The Oxford Guide to Etymology, Oxford, OUP.

Esper, E. 1973. Analogy and association in linguistics and psychology, Athens, The University of Georgia Press.

Evans, N. 2011. Semantic typology, in The Oxford Handbook of linguistics typology, ed. J., Song, Oxford, OUP. 504-533.

Fehling, D. 1956. Varro und die grammatische Lehre von der Analogie und der Flexion I, «Glotta», 35. 214-70.

Fehling, D. 1957. Varro und die grammatische Lehre von der Analogie und der Flexion II, «Glotta», 36. 48-100.

Fleisch, H. 1994. Arabic linguistics, in G. Lepschy (ed.), Volume I. 164-184.

Gilula, M. 1982. An Egyptian etymology of the name of Horus?, «The Journal of Egyptian Archeology», 68. 259-265.

Haiman, J. 1985. Natural syntax. Iconicity and erosion, Cambridge, CUP.

Hock, H. ${ }^{2} 1991$. Principles of historical linguistics, Berlin/New York, De Gruyter. 
Jacobsen, Th. 1974. Very ancient linguistics: Babylonian grammatical texts, in Studies in the history of linguistics. Traditions and paradigms, ed. D. Hymes, Bloomington, Indiana University Press. 41-62.

Johnson, J. 1994. Ancient Egyptian linguistics, in G., Lepschy (ed.) Volume I. 63-76. Jungen, O. and H., Lohnstein. 2006. Einführung in die Grammatiktheorie, München, Fink. Katz, J. 2010. Etymology, in The classical tradition, ed. A. Grafton, G. Most and S. Settis, Cambridge (MA)/London, Harvard University Press. 342-45.

Lallot, J. 1991. ЕТYMОЛОГIA: l'étymologie en Grèce ancienne d'Homère aux grammairiens alexandrins, in J.-P. Chambon and G. Lüdi (eds.). 135-148.

Langendoen, T. 1966. A note on the linguistic theory of M. Terentius Varro, «Foundations of Language», 2. 33-36.

Leiss, E. 2009. Sprachphilosophie, Berlin / New York, de Gruyter.

Lepschy, G. (ed.) 1994. History of linguistics, London/New York, Longman.

Lersch, L. 1838. Die Sprachphilosophie der Alten, Bonn, König.

Loprieno, A. 1995. Ancient Egyptian. A linguistic introduction, Cambridge, CUP.

Lyons, J. 1977. Semantics, Cambridge, CUP.

Malkiel, Y. 1993. Etymology, Cambridge, CUP.

Matthews, P. 1994. Greek and Latin linguistics, in G. Lepschy (ed.), Volume II. 1-133.

Meillet, A. 1931. Caractère secondaire du type thématique indo-européen, «Bulletin de la Societé de Linguistique de Paris», 32. 194-203.

Nathan, T. and Hounkpatin, L. 1993. Oro Lè - la puissance de la parole en psychoanalyse et dans les systèmes thérapeutiques yorubas, «Revue Française de Psychanalyse», 57. 787-805.

Newen, A. and Schrenk, M. 2008. Einführung in die Sprachphilosophie, Darmstadt, Wissenschaftliche Buchgesellschaft.

Noegel, S. 2010. Sign, sign, everywhere a sign: script, power, and interpretation in the Ancient Near East, in Divination and interpretation of signs in the ancient world, ed.

A. Annus, Chicago, The University of Chicago Press. 143-162.

Okasha, S. 2002. Philosophy of science. A very short introduction, Oxford, OUP.

Pfaffel, W. 1981. Quartus gradus etymologiae. Untersuchungen zur Etymologie Varros in 'De Lingua Latina', Königstein, Hain.

Pisani, V. 1967. L'etimologia, Brescia, Paideia.

Reichler-Béguelin, M.-J. 1995. Saussure et l'étymologie populaire, «Lynx», 7. 2-12.

Reiner, E. 1994. Akkadian linguistics, in G. Lepschy (ed.), Volume I. 87-96.

Saussure, F. de 1916. Cours de linguistique générale, ed. C. Bally and A. Sechehaye, Lausanne/Paris, Payot.

Sedley, D. 2003. Plato's Cratylus, Cambridge, CUP.

Schmitt, R. (ed.) 1977: Etymologie, Darmstadt, Wissenschaftliche Buchgesellschaft.

Schleicher, A. 1873. Die darwinsche Theorie und die Sprachwissenschaft: offenes Sendschreiben an Herrn Dr. Ernest Hackel.

Taylor, D. 1975. Declinatio: a study of the linguistic theory of Marcus Terentius Varro, Amsterdam, Benjamins.

Taylor, D. 1977. Varro's mathematical models of inflection, «Transactions of the American Philological Association», 107. 313-323.

Taylor, D. 1996. Varro - De Lingua Latina X, Amsterdam, Benjamins.

Vendryes, J. 1955. Sur l'étymologie croisée, «Bulletin de la Société de Linguistique de Paris», 51. 1-8.

Versteegh, K. 1997. The Arabic linguistic tradition, London/New York, Routledge. 
Viti, C. 2014. On etymology and lexical categorization in the ancient Indo-European languages, «International Journal of Diachronic Linguistics and Linguistic Reconstruction», 11. 1-31.

Walde, A. and Pokorny, J. 1927-1932. Vergleichendes Wörterbuch der indogermanischen Sprachen, Bern \& München, Francke.

Watkins, C. 1990. Etymologies, equations, and comparanda: types and values, and criteria for judgment, in Linguistic change and reconstruction methodology, ed. Ph. Baldi, Berlin/New York, de Gruyter. 289-304. 
Bereitgestellt von | UZH Hauptbibliothek / Zentralbibliothek Zürich Angemeldet Heruntergeladen am | 09.01.17 10:30 